JANUARY 2004

\title{
TRUNCATIONS DRIVEN BY CONSTRAINTS: CONSISTENCY AND CONDITIONS FOR CORRECT UPLIFTINGS
}

\author{
Josep M. Pons ${ }^{a}$ And Pere Talavera ${ }^{b}$ \\ ${ }^{a}$ Departament d'Estructura $i$ Constituents de la Matèria, Universitat de Barcelona, \\ Diagonal 647, E-08028 Barcelona, Spain. \\ ${ }^{b}$ Departament de Física i Enginyeria Nuclear, Universitat Politècnica de Catalunya, \\ Jordi Girona 1-3, E-08034 Barcelona, Spain.
}

\begin{abstract}
We discuss the mechanism of truncations driven by the imposition of constraints. We show how the consistency of such truncations is controlled, and give general theorems that establish conditions for the correct uplifting of solutions. We show in some particular examples how one can get correct upliftings from $7 d$ supergravities to $10 d$ type IIB supergravity, even in cases when the truncation is not initially consistent by its own.
\end{abstract}

E-mail: pons@ecm.ub.es, pere.talavera@upc.es 


\section{Contents}

1 Motivation and general set up 1

2 Second-type truncations. Dynamical consequences of constraints 4

3 Applications: general structure $\quad 6$

3.1 Setting the framework . . . . . . . . . . . . . . . . 7

3.2 Truncation from $10 d$ to $7 d \ldots \ldots \ldots$. . . . . . . . . . . . 8

3.2.1 First step: pure dimensional reduction . . . . . . . . . . . . . . 8

3.2.2 Second step: Truncation driven by constraints . . . . . . . . . . . . . 9

4 Applications: specific models 10

4.1 IIB NS-5 branes . . . . . . . . . . . . . . . . . . . . . . . . 11

4.2 D5-branes wrapping $S^{2} \ldots \ldots \ldots \ldots$

5 Conclusions $\quad 14$

A Proof of the theorems

A.1 Notation and preliminaries . . . . . . . . . . . . . . . . 15

A.2 Proof of theorem 1 . . . . . . . . . . . . . . . . . . 18

A.2.1 Final remarks . . . . . . . . . . . . . . . . 20

A.3 Proof of theorem $2 \ldots \ldots \ldots \ldots \ldots$

\section{Motivation AND GENERAL SET UP}

In recent years, owing to the success of the AdS/CFT correspondence, there has been intense work in trying to extend this correspondence to supergravity backgrounds preserving less than maximal supersymmetry. One way of achieving the construction of such models is to derive them as upliftings of solution of lower dimensional theories, related to the higher dimensional ones by a process of truncation. The physical motivation in terms of the ground state can be seen as follows: gravitational theories in higher dimension can lead after spontaneous compactification to theories that accommodate the Standard Model or the like at $4 d$ $[1]^{1}$. Thus the higher dimensional theory can be seen as a "unification" theory. Even though

\footnotetext{
${ }^{1} \mathrm{~A}$ possible way to determine the ground state is by an uplifting procedure.
} 
the approach is appealing it has non-trivial difficulties and after some decades of work is still lacking a general procedure.

The scope of this paper is not to present a new procedure of truncation nor a new solution for supergravity backgrounds preserving some amount of supersymmetry. Our only concern here is just to systematize a general procedure for obtaining upliftable solutions for certain cases of truncations. Several results in this respect are presented under the form of theorems. In addition, we give some examples of models currently used in the literature to enlighten their construction and the consistency of the truncations involved.

Before proceeding let us define briefly the concept of truncation, which has been already used above. This definition will be expanded later on. This word, truncation, is commonly used with two different, though related, meanings. Given an action functional on a manifold describing a theory one says, grosso modo, that it has undergone a truncation if: i) there has been a reduction of the space-time coordinates -that is, a dimensional reduction-, or ii) the number of d.o.f. in the theory (fields or field components) has been reduced.

In the remainder we shall describe briefly these two types of truncation.

i) First-type truncation, or pure dimensional reduction. In the most simplest setting the dimension of the space-time is reduced by considering the description, through a new action principle, of the subset of solutions of the original equations of motion (e.o.m.) that share some Killing symmetries, while keeping unchanged the number of d.o.f. attached to every space-time point. In more general terms, dimensional reductions proceed along these two main categories

- The reduction is performed via a group manifold of independent Killing symmetries. Its actual form is dictated by the isometry group of the field configurations. The reduction is consistent ${ }^{2}$ as long as the tracelessness condition is fulfilled by the structure constants of the Lie group [2, 3, 4].

- The reduction is done on a coset space. In this case the Killing vectors are no longer independent. Despite many efforts we believe that the state of the art nowadays does not provide yet with a systematic understanding of this kind of truncation procedure and its consistency. For a review up to the 80's see for instance [5] (and references therein). Recent developments can be found in [6, 7]. In this case the reduction is performed under some symmetry considerations but

\footnotetext{
${ }^{2}$ The concept of consistency for a truncation will be defined below.
} 
usually for its own consistency needs to be mixed with a second-type truncation (see below) on the field configurations.

ii) Second-type truncation. It consists in the introduction of constraints that produce a further reduction of the number of independent fields -or field components- defining the theory. We shall only consider constraints in configuration space. Let us mention that, as will be shown below, the consistency of this type of truncations is model dependent and ought to be considered in a case a case basis.

As has been mentioned before these two types of truncation are usually applied altogether under the common concept of dimensional reduction, but in order to give some insight on the model construction we think it is very convenient to maintain a clear distinction between them. Notice, however, that in both cases we are producing a truncation in the field content of the theory, either because an infinity of Kaluza-Klein modes are eliminated in the dimensional reduction process or because some field components become redundant due to the presence of constraints.

The results presented above concerning the consistency of a truncation are purely classical. One can of course use these results within a strategy to obtain solutions of the higher dimensional theory by uplifting from solutions, perhaps easier to find, of the lower dimensional one. These solutions can be for instance candidates for a ground state.

A different and in some sense complementary perspective is that of compactification [8, 9]. It already considers a classical solution, for instance, a vacuum undergoing spontaneous compactification (as a spontaneous symmetry breaking) that exhibits a space-time with a compact component, and formulates a quantum field theory on this background. An expansion in modes (Kaluza-Klein) over the compact space may allow to keep only the massless modes in an effective field theory sense, thus ending up with an effective truncation of the theory.

Before proceeding we shall state the concept of consistent truncation. If we denote generically as $\Phi$ the original fields, and $\tilde{\Phi}$ the remaining fields after the truncation, a definite prescription allows the reconstruction of the configuration of the former from that of the latter. At the level of the variational principle there is a natural map from an initial Lagrangian $\mathcal{L}^{(d+n)}$ into a new one $\mathcal{L}^{(d)}$, perhaps with lower dimensionality -when $n>0$-, perhaps with fewer d.o.f. ,

$$
\mathcal{L}^{(d+n)}(\Phi(\mathbf{x}, \mathbf{y})) \rightarrow \mathcal{L}^{(d)}(\tilde{\Phi}(\mathbf{x}))
$$


with $\Phi(\tilde{\Phi})$ any field of the original (final) theory. Then a truncation, be it first-type, secondtype or mixed, is consistent if the solutions of the equations of motion for the reduced Lagrangian $\mathcal{L}^{(d)}$ are still solutions of the e.o.m. for the original Lagrangian $\mathcal{L}^{(d+n)}$.

The issue of consistency for second-type truncations was examined in [4] but the answer given there was incomplete, for, although some particular cases were worked out, no general result was given concerning the effect of the introduction of constraints. It is our purpose to amend here this incompleteness.

\section{Second-type truncations. Dynamical Consequences of CONSTRAINTS}

In this section we shall give general results on second-type truncations. The introduction of constraints (denoted by $f^{A}(\Phi)$, where $\Phi$ represents generically the fields in the theory) on the configuration space of a field theory may have two distinct effects, according to how they affect the gauge freedom of the theory. They can act as gauge-fixing constraints, thus restricting the gauge freedom, or they may respect the gauge freedom. The first case has been dealt with in [10] and will not be discussed further. The second case will be our concern here.

The reduced theory is obtained after inserting the conditions $f^{A}=0$ into the original Lagrangian. The typical way to proceed is to find an independent set of fields, denoted generically as $\tilde{\Phi}$, out of the original set $\Phi$, such that the constraints $f^{A}=0$ can be equivalently written as $\Phi=F(\tilde{\Phi})$. We represent it as

$$
\mathcal{L} \longrightarrow \mathcal{L}_{\mathrm{R}}:=(\mathcal{L})_{f}{ }^{A}=0 \quad \text { with } \quad \mathcal{L}_{\mathrm{R}}[\tilde{\Phi}]:=\mathcal{L}[F(\tilde{\Phi})]
$$

Using the Dirac-Bergman approach to deal with the formalism of gauge theories [11, 12, we observe that in the canonical formalism there will exist some primary constraints $\phi_{\mu}$ that, unlike the constraints $f^{A}=0$ introduced ad hoc, are inherently born to the formalism. In order to respect the gauge freedom, we shall assume that the truncation constraints are firstclass with respect to the primary constraints. Technically this corresponds to the Poisson bracket

$$
\left\{\phi_{\mu}, f^{A}\right\} \approx 0
$$

i.e., $\left\{\phi_{\mu}, f^{A}\right\}$ vanishes on the surface defined by $\phi_{\mu}=0, f^{A}=0$.

With this assumption the following theoretical result can be formulated 
Theorem 1: The e.o.m. of $\mathcal{L}$, in addition to the condition to satisfy the constraints $f^{A}=0$, are equivalent to the e.o.m. of $\mathcal{L}_{\mathrm{R}}$ plus the condition to satisfy some-secondary-constraints $\chi^{A}=0$.

We shall only sketch the proof of the theorem, leaving the complete, technical proof, together with the precise construction of the constraints $\chi^{A}$, to the appendix. The condition for the dynamics defined by the Lagrangian $\mathcal{L}$ to be compatible with the constraints $f^{A}=0$, which is a tangency condition, imposes in general the existence of new, secondary, constraints. When the constraints $f^{A}=0$ are plugged within these secondary constraints we get the constraints $\chi^{A}=0$. Then one can prove that the dynamics defined by $\mathcal{L}$ on $f^{A}=0$ coincides with the dynamics defined by $\mathcal{L}_{\mathrm{R}}$ on $\chi^{A}=0$.

Notice that this theorem guarantees that any solution of the reduced theory $\mathcal{L}_{\mathrm{R}}$ satisfying the secondary constraints $\chi^{A}=0$ can be uplifted to a solution of the original theory $\mathcal{L}$ satisfying $f^{A}=0$. On the other hand, if a solution of $\mathcal{L}_{\mathrm{R}}$ does not satisfy the constraints $\chi^{A}=0$ it will not be upliftable to a solution of the original theory $\mathcal{L}$.

The presence of these new constraints $\chi^{A}=0$ in the reduced theory is bound to make, in principle, the truncation inconsistent, except for some exceptional cases where the restrictions $\chi^{A}=0$ are void or already included in the e.o.m. of the reduced Lagrangian. But we have just opened the way for a stabilization mechanism: we can start again with the new Lagrangian $\mathcal{L}_{\mathrm{R}}$ and the new constraints $\chi^{A}=0$ and perform another truncation of the same type. Several possibilities are open when we try to run this stabilization mechanism again.

i) It might be that some of the constraints $\chi^{A}$ are indeed gauge fixing constraints. As we have said before, their effect on the theory, see [10], is quite different from the one just examined and must be dealt with accordingly.

ii) Some of the constraints $\chi^{A}$ may not be holonomic, so the process of reducing the d.o.f. may become more sophisticated than just eliminating fields or field components in configuration space.

These situations must be worked in a case by case basis.

iii) All the constraints $\chi^{A}$ are holonomic, hence the above theorem applies, the stabilization mechanism can be run again and the three possibilities we are examining are open for the next step. Let us consider the most favorable case, i.e. the constraints are always holonomic. A possible outcome may be that after a certain number of steps, no new constraints appear. In fact if the algorithm does not stop, since the number of d.o.f. per space-time point is finite, we shall end up with no d.o.f. at all, thus signaling the 
incompatibility of the constraints $f^{A}=0$ with the dynamics derived from the original Lagrangian $\mathcal{L}$.

On the other hand, if the algorithm stops the following proposition can be proven

Proposition: If all constraints, $\chi^{A}$ and their subsequent stabilizations, are holonomic and non-gauge-fixing, and the algorithm stops, the final theory is a consistent truncation of the original one.

The proof is immediate just by examining the last step of the algorithm. In fact this is the case in both of the examples discussed below. This result can also be given a geometric flavor as follows. Consider the tangent space $\mathcal{T} Q$ of a configuration manifold $Q$, and some dynamics defined in it by means of a variational principle. If $\bar{Q}$ is a submanifold of $Q$, such that the dynamics in $\mathcal{T} Q$ is tangent to the submanifold $\mathcal{T} \bar{Q}$, then the variational principle can be directly formulated in $\mathcal{T} \bar{Q}$. This is just the formulation given by the reduced Lagrangian, thus providing with a consistent truncation of the original theory.

Another mechanism to guarantee that a truncation driven by constraints is consistent is provided by symmetry considerations. One can state

Theorem 2: If the constraints $f^{A}$ generate, via the Poisson brackets, a symmetry of the e.o.m., then the truncation is consistent.

The proof is given in the appendix.

\section{Applichtions: General structure}

In order to exemplify the above general results we shall work out explicitly two bosonic solutions characterized by: i) both are solutions of theories obtained as low-energy field theory limits of type-IIB string theory and $i i$ ) the solutions are obtained via uplifting from $7 d$ to $10 d$ after the truncation of the initial theory to $7 d$. As both solutions are related we shall make a common treatment of the first steps in the truncation and uplifting procedure.

Before proceeding to the subject proper, however, we must first discuss briefly some technical details: the space-time manifold we shall consider is constituted by a non-compact 
part times a compact one, $\mathcal{M}_{d} \otimes \mathcal{H}_{n}$. The requirement of compacity ensures the factorization of a finite volume in the action. In the cases we shall develop below, we identify $\mathcal{H}_{n} \equiv S^{3}$, where the $S^{3}$ is considered as the group manifold $S U(2)$. Its isometry group is $S O(4)=$ $S U(2) \times S U(2)$ but we assume that only one of the $S U(2)$ 's is an isometry of the full metric on $\mathcal{M}_{d} \otimes \mathcal{H}_{n}$. In particular we shall tackle two solutions [13, 14] that can be thought as upliftings after a previous truncation of the theory from $10 d$ to $7 d$. In this case the Lie algebra of Killing vectors, $K_{a}=K_{a}^{\alpha}(y) \partial_{\alpha}$, with $\left[K_{a}, K_{b}\right]=C_{a b}^{c} K_{c}$, used in the dimensional reduction, is that of $s u(2)$. The $y^{\alpha}$ 's denote the $S^{3}$ coordinates and will eventually disappear in the truncation. As a matter of notation, the indices $\alpha$ and $a$ run over $1,2,3$, and $\psi^{1}, \theta^{1}, \phi^{1}$ are the parameters of the sphere $S^{3}$. One can construct a basis of left-invariant one-forms, $\omega^{a}=\omega_{\alpha}^{a}(y) d y^{\alpha}$, with $\mathfrak{L}_{K_{a}} \omega^{b}=0$, where $\mathfrak{L}_{K_{a}}$ stands for the Lie derivative with respect to the vector $K_{a}$. In our conventions,

$$
\omega^{1}+i \omega^{2}=\frac{1}{2} e^{-i \psi^{1}}\left(d \theta^{1}+i \sin \theta^{1} d \phi^{1}\right), \quad \omega^{3}=\frac{1}{2}\left(d \psi^{1}+\cos \theta^{1} d \phi^{1}\right) .
$$

This basis satisfies the Cartan-Maurer equations $d \omega^{a}=\frac{1}{2} C_{b c}^{a} \omega^{b} \wedge \omega^{c}$, with the structure constants $C_{b c}^{a}=-2 \epsilon_{a b c}$.

The manifold is also provided with a rank-2 symmetric tensor, the metric field $g$, satisfying the Killing conditions, $\mathfrak{L}_{K_{a}} g=0$. Written in the mixed basis $d x^{\nu}, \omega^{a}$, it takes the form

$$
g=g_{\mu \nu}(x) d x^{\mu} d x^{\nu}+g_{a b}(x)\left(A_{\mu}^{a}(x) d x^{\mu}+\omega^{a}\right)\left(A_{\nu}^{b}(x) d x^{\nu}+\omega^{b}\right) .
$$

Notice that we keep all components of the metric. The Killing conditions make them to depend only on the $x$ variables.

After the first-type truncation the quotient manifold is parametrised by the $x^{\mu}$ coordinates $(\mu=0,1, \ldots, 6)$.

\subsection{Setting the Framework}

For the two solutions at hand the starting point will be the bosonic sector ${ }^{3}$ of type IIB supergravity $(d+n=7+3=10)[15]$

$$
\begin{aligned}
S_{\mathrm{S}}^{(d+n)}= & \frac{1}{2 \kappa_{0}^{2}} \int d^{d} x d^{n} y \sqrt{-\hat{g}}\left\{e^{-2 \hat{\Phi}}\left[\hat{\mathcal{R}}+4 \partial_{\hat{\mu}} \hat{\Phi} \partial_{\hat{\nu}} \hat{\Phi} \hat{g}^{\hat{\mu} \hat{\nu}}-\frac{1}{12}\left(\hat{H}^{(3)}\right)^{2}\right]\right. \\
& \left.-\frac{1}{12}\left(\hat{G}^{(3)}+\hat{C}^{(0)} \hat{H}^{(3)}\right)^{2}-\frac{1}{2} d \hat{C}^{(0)} d \hat{C}^{(0)}-\frac{1}{480} \hat{G}^{(5)} \hat{G}^{(5)}\right\} \\
& +\frac{1}{4 \kappa_{0}^{2}} \int\left(\hat{C}^{(4)}+\frac{1}{2} \hat{B}^{(2)} \hat{C}^{(2)}\right) \hat{G}^{(3)} \hat{H}^{(3)},
\end{aligned}
$$

\footnotetext{
${ }^{3}$ This sector by its own is a consistent truncation of the full theory.
} 
where $\hat{H}^{(3)}:=d \hat{B}^{(2)}$ is the field strength of the NS two-form,

$$
\left(\hat{H}^{(3)}\right)^{2}:=\hat{H}_{\hat{\mu} \hat{\nu} \hat{\rho}} \hat{g}^{\hat{\mu} \hat{\alpha}} \hat{g}^{\hat{\nu} \hat{\beta}} \hat{g}^{\hat{\rho} \hat{\gamma}} \hat{H}_{\hat{\alpha} \hat{\beta} \hat{\gamma}}
$$

and $\hat{G}^{(3)}:=d \hat{C}^{(2)}, \hat{G}^{(5)}:=d \hat{C}^{(4)}+\hat{H}^{(3)} \hat{C}^{(2)}$ are the RR field strengths. In addition $\hat{C}^{(0)}$ stands for the RR scalar field, the axion. For the time being $n$ and $d$ are kept generic. Notice that we can consistently set to zero the RR field strengths and the RR scalar field without any further implication in the theory. This is due to the fact that the appearance of these fields inside the action is quadratic and hence they identically imply a vanishing equation of motion when set to zero. At this early stage, and after substitution of the inverse matrix corresponding to (3.2),

$$
\hat{g}^{\hat{\mu} \hat{\nu}}=\left(\begin{array}{cc}
g^{\mu \nu} & -g^{\mu \nu} A_{\mu}^{a} Y_{a}^{\beta} \\
-g^{\rho \nu} A_{\rho}^{a} Y_{a}^{\alpha} & \left(g^{a b}+g^{\mu \nu} A_{\mu}^{a} A_{\nu}^{b}\right) Y_{a}^{\alpha} Y_{b}^{\beta}
\end{array}\right)
$$

we shall only deal with the expression between squared brackets in (3.6), i.e.

$$
S_{\mathrm{S}}^{(d+n)}=\frac{1}{2 \kappa_{0}^{2}} \int d^{d} x d^{n} y\left|-\hat{g}_{\hat{\mu} \hat{\nu}}\right|^{1 / 2} e^{-2 \hat{\Phi}}\left(\hat{\mathfrak{R}}+4 \partial_{\hat{\mu}} \hat{\Phi} \partial_{\hat{\nu}} \hat{\Phi} \hat{g}^{\hat{\mu} \hat{\nu}}-\frac{1}{12}\left(\hat{H}^{(3)}\right)^{2}\right)
$$

that constitutes already a consistent truncation of type IIB theory. One can verify in addition that it also corresponds to a consistent truncation of type I supergravity.

\subsection{TRUnCATION FROM $10 d$ TO $7 d$}

In the two examples that are examined later, the total truncation can be performed in several steps, combining the two truncations, first-type and second-type, discussed before. Here we shall display the first two steps, common to the two examples, exhibiting already the main features of each type of truncation.

\subsubsection{FIRST STEP: PURE DIMENSIONAL REDUCTION}

The theory is truncated by a pure dimensional reduction from $(d+n)$ dimensions (coordinates $x, y$ ) to $d$ dimensions (coordinates $x$ ), keeping unchanged the number of d.o.f. per space-time point

$$
\mathcal{L}^{(d+n)}\left(\hat{g}_{\hat{\mu} \hat{\nu}}, \hat{\Phi}, \hat{B}_{\hat{\mu} \hat{\nu}}\right) \longrightarrow \mathcal{L}_{\mathrm{R}}^{(d)}\left(g_{\mu \nu}, g_{a b}, A_{\mu}^{a}, \Phi, B_{\mu \nu}, B_{\mu a}, B_{a b}\right) .
$$

The associated Lagrangian density, $\mathcal{L}_{\mathrm{R}}^{(d)}$, is a first-type consistent truncation of $\mathcal{L}^{(d+n)}$ with an expression which deduced from the $(d+n)$-dimensional Einstein frame takes the form 
[2, 16]

$$
\begin{aligned}
\mathcal{L}_{\mathrm{R}}^{(d)}= & \frac{\mathrm{Vol}_{\mathrm{n}}}{2 \kappa_{0}^{2}}\left|-g_{\mu \nu}\right|^{1 / 2}\left|g_{a b}\right|^{1 / 2}\left\{\mathfrak{R}-\frac{1}{4} F^{\mu \nu a} F_{\mu \nu}^{b} g_{a b}+\frac{1}{4} g^{\mu \nu} \mathcal{D}_{\mu} g_{a b} \mathcal{D}_{\nu} g^{a b}\right. \\
& +g^{\mu \nu} \partial_{\mu} \ln \sqrt{g_{a b}} \partial_{\nu} \ln \sqrt{g_{a b}}-\frac{1}{4} C_{b c}^{a}\left[2 C_{a c^{\prime}}^{b} g^{c c^{\prime}}+C_{b^{\prime} c^{\prime}}^{a^{\prime}} g_{a a^{\prime}} g^{b b^{\prime}} g^{c c^{\prime}}\right] \\
& \left.-\frac{1}{2}(\nabla \Phi)^{2}-\frac{1}{12} e^{-\Phi}\left(H^{(3)}\right)^{2}\right\}
\end{aligned}
$$

being $\mathcal{D}_{\mu}$ the covariant derivative for the Yang-Mills connection and $\operatorname{Vol}_{n}=\int|\omega| d^{n} y$. It is not necessary to express the components of the NS-NS gauge field strength. The legitimacy of the procedure is guaranteed by the tracelessness condition, $C_{a b}^{a}=0$. Any solution of the e.o.m. for $\mathcal{L}_{\mathrm{R}}^{(d)}$ can be uplifted to a solution of the e.o.m. for $\mathcal{L}^{(d+n)}$, and vice-versa, any solution of the e.o.m. for $\mathcal{L}^{(d+n)}$ that satisfies the Killing conditions can be obtained as an uplifting of a solution of the e.o.m. for $\mathcal{L}_{\mathrm{R}}^{(d)}$.

Hitherto the space-time dimension has been general, henceforth, and for sake of clarity, we set $d=7, n=3$. The Lie group of Killing symmetries is $S U(2)$.

\subsubsection{Second Step: Truncation Driven By CONStraints}

It consists in truncating the scalar spectrum of the theory. This is a second-type truncation, driven by constraints, and its consistency relies in the findings of sec. 2. We introduce, in the $\mathcal{L}_{\mathrm{R}}^{(7)}$ theory, the constraints ${ }^{4}$

$$
g_{a b} C_{c d}^{a}+g_{a d} C_{c b}^{a}=0
$$

For a simple Lie algebra, where with our conventions the Cartan-Killing constant metric $h_{a b}:=C_{a d}^{c} C_{b c}^{d}$ is a multiple of $\delta_{a b}$, equation (3.8) is equivalent to the more familiar relation

$$
g_{a b}=\varphi \delta_{a b}
$$

for some $\varphi$ that becomes the only remaining scalar field from those originated from the 10dimensional metric. These constraints eliminate all the scalars that carry YM charges and make the internal manifold bi-invariant, under two copies of the simple Lie group. We have

$$
\mathcal{L}_{\mathrm{R}}^{(7)}\left(g_{\mu \nu}, g_{a b}, A_{\mu}^{a}, \Phi, B_{\mu \nu}, B_{\mu a}, B_{a b}\right) \longrightarrow \mathcal{L}_{2 \mathrm{R}}^{(7)}\left(g_{\mu \nu}, \varphi, A_{\mu}^{a}, \Phi, B_{\mu \nu}, B_{\mu a}, B_{a b}\right)
$$

\footnotetext{
${ }^{4}$ To introduce some constraints in a theory means that we look for solutions of the e.o.m. of the theory that in addition satisfy such constraints.
} 
with the r.h.s. given by

$$
\begin{aligned}
\mathcal{L}_{2 \mathrm{R}}^{(7)}= & \frac{\mathrm{Vol}_{3}}{2 \kappa_{0}^{2}}\left|-g_{\mu \nu}\right|^{1 / 2} \varphi^{3 / 2}\left(\mathfrak{R}-\frac{1}{4} \varphi F^{a} F^{b} \delta_{a b}+\frac{3}{2}\left(\frac{\nabla \varphi}{\varphi}\right)^{2}+\frac{6}{\varphi}-\frac{1}{2}(\nabla \Phi)^{2}\right. \\
& \left.-\left.\frac{1}{12} e^{-\Phi}\left(\hat{H}^{(3)}\right)^{2}\right|_{g_{a b}=\varphi \delta_{a b}}\right) .
\end{aligned}
$$

As regards the e.o.m. it turns out that

$$
\begin{aligned}
& \left(\frac{\delta \mathcal{L}_{\mathrm{R}}^{(7)}}{\delta g_{\mu \nu}}\right)_{g_{a b}=\varphi \delta_{a b}}=\frac{\delta \mathcal{L}_{2 \mathrm{R}}^{(7)}}{\delta g_{\mu \nu}}, \\
& \left(\frac{\delta \mathcal{L}_{\mathrm{R}}^{(7)}}{\delta A_{\mu}^{a}}\right)_{g_{a b}=\varphi \delta_{a b}}=\frac{\delta \mathcal{L}_{2 \mathrm{R}}^{(7)}}{\delta A_{\mu}^{a}}, \\
& \left(\frac{\delta \mathcal{L}_{\mathrm{R}}^{(7)}}{\delta g_{a b}}\right)_{g_{a b}=\varphi \delta_{a b}}=\frac{1}{3}\left(\frac{\delta \mathcal{L}_{2 \mathrm{R}}^{(7)}}{\delta \varphi}\right) \delta^{a b}-\left(\frac{\mathrm{Vol}_{3}}{2 \kappa_{0}^{2}}\left|g_{\mu \nu}\right|^{\frac{1}{2}} \varphi^{\frac{3}{2}}\right) \chi^{a b},
\end{aligned}
$$

with

$$
\chi^{a b}:=\frac{1}{4} M^{a b}-\frac{1}{12}\left(M^{c d} \delta_{c d}\right) \delta^{a b}, \quad M^{a b}:=F_{\mu \nu}^{a} F^{\mu \nu b}-\frac{e^{-\Phi}}{\varphi^{2}} H_{c \hat{\mu} \hat{\nu}} H_{d}{ }^{\hat{\mu} \hat{\nu}} \delta^{c a} \delta^{d b} .
$$

At first sight we realize that the truncation is inconsistent because the presence of a term proportional to $\chi^{a b}$ in the r.h.s. (3.11), which may be different from zero. What we have found are just the secondary constraints predicted by our theorem of sec. 2. For a simplified model with the same features see [4]. We must therefore require

$$
\chi^{a b}=0,
$$

on the candidate configurations for an uplifting from the $\mathcal{L}_{2 \mathrm{R}}^{(7)}$ theory to the $\mathcal{L}_{\mathrm{R}}^{(7)}$.

\section{AppliCATIONS: SPECIFIC MODELS}

After showing the general pattern to be followed, we find it worthwhile to work out two specific examples that comply the requirement of being built on semisimple Lie algebras. As we mentioned already in sec. 3 both examples consider basically a truncation of $10 d$ supergravity to $7 d$ with a different content of supersymmetry. 


\subsection{IIB NS-5 BRANES}

Let us consider the consistent bosonic truncation of Type IIB, which in the Einstein frame takes the form

$$
S_{\mathrm{E}}^{(10)}=\frac{1}{2 \kappa_{0}^{2}} \int d^{10} x\left|-\hat{g}_{\hat{\mu} \hat{\nu}}\right|^{1 / 2}\left(\hat{\mathfrak{R}}-\frac{1}{2} \partial_{\hat{\mu}} \hat{\Phi} \partial_{\hat{\nu}} \hat{\Phi} \hat{g}^{\hat{\mu} \hat{\nu}}-\frac{1}{12} e^{-\hat{\Phi}}\left(\hat{H}^{(3)}\right)^{2}\right) .
$$

The reduced Lagrangian can be read of from (3.7).

As is analyzed in [4, and in agreement with the theorem formulated in section sec. 2, the consequence of imposing the constraints (3.8) is the emergence of new, secondary constraints, that take the form (3.12). A strong way to satisfy these new constraints is to impose on the theory a new, drastic second-type truncation:

$$
A_{\mu}^{a}=H_{\mu \nu \sigma}=H_{\mu \nu a}=H_{\mu a b}=0,
$$

which is trivially consistent because the Lagrangian is quadratic in these field components that are set to zero.

After imposition of this new truncation we obtain a newly reduced Lagrangian which is already a consistent truncation of Type IIB supergravity, with no constraints attached,

$$
\mathcal{L}_{3 \mathrm{R}}^{(7)}=\frac{\mathrm{Vol}_{3}}{2 \kappa_{0}^{2}}\left|-g_{\mu \nu}\right|^{1 / 2} \varphi^{3 / 2}\left(\mathfrak{R}+\frac{3}{2}\left(\frac{\nabla \varphi}{\varphi}\right)^{2}+\frac{6}{\varphi}-\frac{1}{2}(\nabla \Phi)^{2}-\frac{1}{12} e^{-\Phi} \varphi^{-3}(H)^{2}\right),
$$

where $(H)^{2}=H_{a b c} \delta^{a a^{\prime}} \delta^{b b^{\prime}} \delta^{c c^{\prime}} H_{a^{\prime} b^{\prime} c^{\prime}}$.

Moving to the Einstein frame, and defining $\varphi=e^{\Psi}$, we re-express this theory as

$$
\mathcal{L}_{\mathrm{E}}^{(7)}=\frac{\mathrm{Vol}_{3}}{2 \kappa_{0}^{2}}\left|-g_{\mu \nu}\right|^{1 / 2}\left(\mathfrak{R}-\frac{6}{5}(\nabla \Psi)^{2}+6 e^{-\frac{8}{5} \Psi}-\frac{1}{2}(\nabla \Phi)^{2}-\frac{1}{12} e^{-\Phi} e^{-\frac{18}{5} \Psi}(H)^{2}\right) .
$$

Notice that the object $H_{a b c}$, completely antisymmetric, is just the product of a $7 d$ scalar times $\epsilon_{a b c}$. But since, when uplifted to $10 d$, it is interpreted as giving rise to the components of a three-from field strength, $H=H_{a b c} \omega^{a} \wedge \omega^{b} \wedge \omega^{c}$, in order for $H$ to be a closed form satisfying the Killing conditions, this scalar must be simply a constant.

This Lagrangian can undergo a new second-type truncation by linking the two scalars, $\Phi$ and $\Psi$. Let us consider the new constraint

$$
\chi:=\Psi+\lambda \Phi=0,
$$

where the constant parameter $\lambda$ will be determined below by requiring the truncation to be eventually consistent. The e.o.m. for $\Phi$ and $\Psi$ are

$$
\begin{aligned}
\triangle \Phi+\frac{1}{12} e^{-\Phi} e^{-\frac{18}{5} \Psi}(H)^{2} & =0, \\
\triangle \Psi+\frac{1}{8} e^{-\Phi} e^{-\frac{18}{5} \Psi}(H)^{2}-4 e^{-\frac{8}{5} \Psi} & =0,
\end{aligned}
$$


and therefore

$$
\triangle \chi+\left(\frac{3+2 \lambda}{24}\right) e^{-\Phi} e^{-\frac{18}{5} \Psi}(H)^{2}-4 e^{-\frac{8}{5} \Psi}=0 .
$$

Since the constraint $\chi$ is set to zero, the secondary constraint is isolated as

$$
(3+2 \lambda) e^{-\Phi-2 \Psi}(H)^{2}=96 .
$$

We can make the choice $\lambda=\frac{1}{2}$. This makes the exponent in the last expression $-\frac{1}{2} \chi$, which is zero because we are implementing $\chi=0$. We end up with the new constraint

$$
(H)^{2}=24
$$

or, equivalently,

$$
H_{a b c}= \pm 2 \epsilon_{a b c} .
$$

This last constraint is in fact very adequate, because we have argued before that the scalar in $H_{a b c}$ must be a constant. Note that any other choice, $\lambda \neq \frac{1}{2}$, would have led to inconsistencies.

One can check that the new constraint (4.4) is compatible with the dynamics without the appearance of new constraints. We have therefore proved that implementing both constraints, $\Psi=-\frac{1}{2} \Phi$ and $H_{a b c}= \pm 2 \epsilon_{a b c}$, directly into the Lagrangian makes the new truncation still consistent. The final Lagrangian is

$$
\mathcal{L}_{\mathrm{E}}^{(7)}=\frac{\mathrm{Vol}_{3}}{2 \kappa_{0}^{2}}\left|-g_{\mu \nu}\right|^{1 / 2}\left(\mathfrak{R}-\frac{4}{5}(\nabla \Phi)^{2}+4 e^{\frac{4}{5} \Phi}\right)
$$

and our construction allows us to assert that this Lagrangian is a consistent truncation of Type IIB supergravity. Any solution of (4.5) can be uplifted to a solution of Type IIB. Notice that in the process of uplifting from $7 d$ to $10 d$ a field strength $H=2 \epsilon_{a b c} \omega^{a} \wedge \omega^{b} \wedge \omega^{c}$ will appear, together with the required components for the $10 d$ metric.

The Lagrangian (4.5) belongs to an interesting family of Lagrangians, one for each spacetime dimension $d>2$, with a metric and a scalar field,

$$
\mathcal{L}_{\mathrm{E}}^{(d)}=\text { const. }\left|-g_{\mu \nu}\right|^{1 / 2}\left(\mathfrak{R}-\frac{4}{d-2}(\nabla \Phi)^{2}+4 e^{\frac{4}{d-2} \Phi}\right),
$$

which are related among themselves by toroidal (i.e., Abelian) consistent truncations. In fact, every single truncation includes $i$ ) a one-dimensional reduction, ii) a second-type truncation to get rid of a Maxwell field, iii) another second-type truncation to link the scalar mode coming from the reduction of the metric with the dilaton, and $i v$ ) a conformal redefinition of the metric in order to stay in the Einstein frame. It is easy to verify that the following conformally flat configuration

$$
\begin{aligned}
g_{\mu \nu}^{(d)} & =e^{\frac{4}{d-2} \rho} \eta_{\mu \nu}^{(d)} \\
\Phi & =-\rho
\end{aligned}
$$


(where $\eta_{\mu \nu}^{(d)}$ is the $d$-dimensional Minkowski metric and $\rho$ is a spatial-coordinate) is a solution of the theory ${ }^{5}$. In fact these solutions for different dimensions are all connected by upliftings. When uplifted from $7 d$ to $10 d$ by use of the $s u(2)$ Killing algebra we obtain a solution of Type IIB that, in Einstein frame, takes the form

$$
\begin{aligned}
\left(d s^{(10)}\right)^{2} & =e^{\frac{1}{2} \rho}\left(d s^{2}\left(\mathbb{E}^{(1,6)}\right)+d \rho^{2}+\sum_{a=1}^{3} \omega^{a} \otimes \omega^{a}\right), \\
\Phi & =-\rho \\
H & =2 \epsilon_{a b c} \omega^{a} \wedge \omega^{b} \wedge \omega^{c} .
\end{aligned}
$$

This is the well known solution describing an extremal Dp-brane [13].

\subsection{D5-BRANES WRAPPING $S^{2}$}

As a second exemplification of the theorem we present a much less straightforward model, which also describes a solution of (4.1). This model describes 5-branes wrapping a two cycle and is dual in the infrared to $\mathcal{N}=1$ super Yang-Mills [17]. It has also been constructed without relying on upliftings in [18, 19, 20]. The first-type truncation follows the same lines as in the previous example, thus the model under consideration will result from uplifting a solution of (3.7). It contains a nonzero NS-NS two-form field given generically by

$$
H^{(3)}=d B^{(2)}=2\left(\omega^{1}+A^{1}\right) \wedge\left(\omega^{2}+A^{2}\right) \wedge\left(\omega^{3}+A^{3}\right)+\sum_{a=1}^{3} F^{a} \wedge\left(\omega^{a}+A^{a}\right)
$$

where $\omega_{i}$ are given in (3.1) and the components of the $S U(2)$ YM potential $A^{a}$ are ${ }^{6}$

$$
A^{1}=\frac{1}{2} a(\rho) d \theta, \quad A^{2}=-\frac{1}{2} a(\rho) \sin \theta d \varphi, \quad A^{3}=\frac{1}{2} \cos \theta d \varphi .
$$

The complete display of the model includes the metric, which in the string frame reads

$$
\left(d s^{(10)}\right)^{2}=d s^{2}\left(\mathbb{E}^{(1,3)}\right)+d \rho^{2}+e^{2 g(\rho)} d \Omega_{2}^{2}+\sum_{a=1}^{3}\left(\omega^{a}+A^{a}\right)^{2},
$$

and the dilaton field,

$$
e^{2 \hat{\phi}(\rho)}=\frac{2 e^{g(\rho)}}{\sinh 2 \rho} .
$$

\footnotetext{
${ }^{5}$ The factor of 4 in the potential term in (4.6) can be generalized to an arbitrary $\beta>0$. Then $\Phi$ changes to $\Phi=-\frac{\sqrt{\beta}}{2} \rho$.

${ }^{6}$ We shall share the definitions of [21] with a value of their parameter $\lambda=-1$.
} 
The functions $a(\rho)$ and $g(\rho)$ remain to be determined. These were first obtained in a $7 d$ context in [14, 22],

$$
a(\rho):=\frac{2 \rho}{\sinh 2 \rho},
$$

and

$$
e^{2 g(\rho)}:=\rho \operatorname{coth} 2 \rho-\frac{\rho^{2}}{\sinh ^{2} 2 \rho}-\frac{1}{4} .
$$

Notice that this last function is highly nontrivial and it runs on the $\rho$ direction; this fact invalidates the premises of [23], and hence allows in principle for a dimensional reduction together with the elimination of degrees of freedom.

Let us summarize the present status. We have on one hand a solution of a $7 d$ supergravity. In addition, there is a set of constraints, (3.12), that, according to the theorem in sec. 2 , play the role of conditions for the uplifting. That is, the $7 d$ solution will be upliftable to a solution of the $10 d$ theory if, and only if, it satisfies (3.12).

The satisfaction of (3.12) is guaranteed by the structure of (4.9), independently of the explicit form of $a(\rho)$ and $g(\rho)$, provided that $\varphi^{2}=e^{-\Phi}$ in the 10d Einstein frame-corresponding to $\varphi=1$ in the string frame-. As a consequence, the $7 \mathrm{~d}$ solution of [14, 22], which has been already displayed in the string frame, is upliftable to $10 \mathrm{~d}$ if the constraint $\varphi=1$ is fulfilled. This condition is indeed satisfied by (4.11), and therefore we conclude that the $7 d$ solution (4.9) 4.14) is upliftable to a solution of Type IIB.

Let us mention that, as in the previous example, if one starts with the right set of constraints [6], the reduction algorithm can be applied several times, as described in sec. 2. In this case one can end up with a consistent truncation of type IIB which still exhibits a non-trivial YM gauge potential [6, 24].

\section{Conclusions}

Despite the big amount of recent literature concerning the truncation and uplifting procedures we find compelling to revise in a original way some models that are commonly used in the context, or in generalizations, of the AdS/CFT duality. In this respect we formulate in a systematic manner the necessary and sufficient conditions, for the construction of supergravity models via uplifting procedures. For this purpose we provide with some theoretical results that stablish under which conditions a truncation of a theory, with holonomic constraints, can lead to consistent upliftable solutions. 
We disentangle in a neat fashion two different steps in the truncation procedure. A first one deals solely with dimensional reduction and a second with the elimination of d.o.f. in configuration space. While the former has been considered elsewhere [4] in the case of quotienting out a group manifold, we have concentrated here in elucidating the consequences of the latter. Is it clear that the first and unavoidable consequence of eliminating some d.o.f. is the existence of classical constraints that have to be satisfied. The theorem in sec. 2 establishes that the theory defined with the remaining d.o.f. must in general be supplemented with secondary constraints. If that is the case the truncation is not consistent by its own. Anyhow correct upliftings can be obtained as long as we consider solutions of the reduced theory that satisfy these new constraints. In the case that the new constraints are defined in configuration space the truncation procedure can be applied again. In this way it is possible to end up with a consistent truncation when no more constraints appear at a given stage.

Our approach have been applied in two models. The first one for its simplicity is just a plain illustration of the different steps, while the second is the only known non-conformal, $\mathcal{N}=1$ SYM supergravity solution.

\section{Acknowledgments}

We thank Carlos Núñez for suggestions and discussions and Arkady Tseytlin for criticism. The work of J. M. P. is partially supported by MCYT FPA, 2001-3598, CIRIT, GC 2001SGR00065, and HPRN-CT-2000-00131.

\section{A. Proof of the theOREMS}

Second type truncations are characterized by the elimination of degrees of freedom per spacetime point. Here we shall consider the case when the constraints that reduce the degrees of freedom are holonomic, that is, when they constrain the variables, fields, in configuration space.

\section{A.1. Notation AND PRELIMINARIES}

For the sake of simplicity in the exposition, we shall use in this section the language of mechanics. The analogous results for field theories are straightforwardly obtained by making use of De Witt's compact notation [?], in which the labels for our objects can represent not only discrete but continuous indices as well. As a matter of notation the evolutionary parameter - the time- is denoted by $t$, and the configuration-space variables are $q^{i}$. With the 
appropriate change of variables we can explicitate the constraints as some of the coordinates. This means that we can take $q^{i}=\left(q^{A}, q^{a}\right)$ with the constraints represented by $q^{A}=0$. The remaining variables $q^{a}$ are the variables for the reduced configuration space.

Consider a Lagrangian $L\left(q^{i}, \dot{q}^{i}\right)$. Plugging the constraints $q^{A}=0, \dot{q}^{A}=0$, into it yields the reduced Lagrangian $L_{\mathrm{R}}\left(q^{a}, \dot{q}^{a}\right)=L\left(q^{A}=0, q^{a}, \dot{q}^{A}=0, \dot{q}^{a}\right)$. The original e.o.m. are

$$
[L]_{i}=\alpha_{i}-W_{i j} \ddot{q}^{j}=0,
$$

where

$$
W_{i j}:=\frac{\partial^{2} L}{\partial \dot{q}^{i} \partial \dot{q}^{j}},
$$

is the Hessian matrix for $L$ w.r.t. the velocities and $\alpha_{i}:=\frac{\partial L}{\partial q^{i}}-\frac{\partial^{2} L}{\partial \dot{q}^{2} \partial q^{j}} \dot{q}^{j}$.

When the reduction, $q^{A}=0, \dot{q}^{A}=0, \ddot{q}^{A}=0$, is implemented at the level of the e.o.m. we get, for $i=a$,

$$
\left([L]_{a}\right)_{\mathrm{R}}=\left(\alpha_{a}\right)_{\mathrm{R}}-\left(W_{a b}\right)_{\mathrm{R}} \ddot{q}^{b}=\tilde{\alpha}_{a}-\tilde{W}_{a b} \ddot{q}^{b}=\left[L_{\mathrm{R}}\right]_{a}=0,
$$

where

$$
\tilde{\alpha}_{a}:=\frac{\partial L_{\mathrm{R}}}{\partial q^{a}}-\frac{\partial^{2} L_{\mathrm{R}}}{\partial \dot{q}^{a} \partial q^{b}} \dot{q}^{b},
$$

and $\tilde{W}_{a b}$ is the Hessian matrix for $L_{\mathrm{R}}$ (tildes quantities will correspond to the reduced formalism, derived from $L_{\mathrm{R}}$ ). In addition, for $i=A$

$$
\left([L]_{A}\right)_{\mathrm{R}}=\left(\alpha_{A}\right)_{\mathrm{R}}-\left(W_{A b}\right)_{\mathrm{R}} \ddot{q}^{b}=0 .
$$

An immediate consequence is that this kind of reduction will generally be inconsistent because the reduced Lagrangian only produces a part of the reduced e.o.m.

$$
\left\{\begin{array}{c}
{[L]_{i}=0} \\
q^{A}=0
\end{array}\right\} \Longleftrightarrow\left([L]_{i}\right)_{\mathrm{R}}=0 \Longleftrightarrow\left\{\begin{array}{c}
{\left[L_{\mathrm{R}}\right]_{a}=0} \\
\left([L]_{A}\right)_{\mathrm{R}}=0
\end{array}\right\}
$$

Notice that the remaining part, $\left([L]_{A}\right)_{\mathrm{R}}=0$, which is not generated by $L_{\mathrm{R}}$, seems to contain in general second derivatives of the variables. But, as we shall see this can be avoided. It is obviously so for theories defined with Lagrangians that are regular, that is, when their Hessian matrix is regular. In such case one could have isolated $\ddot{q}^{A}$ from the e.o.m. $[L]_{i}=0$, that is, $\ddot{q}^{A}=\left(W^{-1}\right)^{A i} \alpha_{i}$, and then implement $q^{A}=0, \dot{q}^{A}=0, \ddot{q}^{A}=0$ on this last relation. This gives

$$
\left(W^{-1} \alpha\right)_{\mathrm{R}}^{A}:=\left(\left(W^{-1}\right)^{A i} \alpha_{i}\right)_{\mathrm{R}}=0,
$$


as new constraints, depending only on $q^{a}, \dot{q}^{a}$. Therefore, for theories defined with regular Lagrangians, the following equivalence holds

$$
\left([L]_{i}\right)_{\mathrm{R}}=0 \Longleftrightarrow\left\{\begin{array}{c}
{\left[L_{\mathrm{R}}\right]_{a}=0} \\
\left(W^{-1} \alpha\right)_{\mathrm{R}}^{A}=0
\end{array}\right\}
$$

Our cases of interest, though, are theories allowing for gauge freedom, and this implies that the Hessian matrix of our Lagrangians must be singular. In such case, the constraints introduced by way of the second-type truncation can be classified according to as to whether they restrict or not the gauge freedom. We shall address the case when the truncation constraints do not restrict the gauge freedom. In order to express this condition, it is convenient to write the original e.o.m. in an equivalent form. To do so one must consider a basis, $\gamma_{\mu}^{i}$, of the null vectors of the Hessian matrix, that satisfy, by definition,

$$
\gamma_{\mu}^{i} W_{i j}=0
$$

It is obvious that the solutions of the e.o.m. (A.1) must satisfy the primary constraints

$$
\alpha \gamma_{\mu}:=\alpha_{i} \gamma_{\mu}^{i}=0
$$

Next consider that although the matrix $W_{i j}$ is not invertible, there exist -non-uniqueobjects $M^{i j}$ (symmetric) and $\sigma_{i}^{\mu}$ such that

$$
W_{k j} M^{j i}+\gamma_{\mu}^{i} \sigma_{k}^{\mu}=\delta_{k}^{i}
$$

We can express the dynamical vector field $\mathbf{X}$ that generates the solution trajectories out of some initial conditions. Saturating (A.3) with $\alpha_{i}$, and taking into account the primary constraints, we end up with

$$
W_{k j}\left(M^{j i} \alpha_{i}\right)-\alpha_{k} \approx 0
$$

where we have borrowed Dirac's notation of weak equalities for those equalities that are satisfied on the constraints' surface.

Comparison of (A.4) with (A.1) allows to isolate $\ddot{q}^{j}$ with its inherent gauge ambiguity:

$$
\ddot{q}^{j}=M^{j i} \alpha_{i}+\eta^{\mu} \gamma_{\mu}^{j}
$$

where $\eta^{\mu}$ are a set of arbitrary functions that essentially reflect the existence of gauge freedom in the theory.

Therefore the dynamical vector field is expressed as

$$
\mathbf{X}:=\dot{q}^{i} \frac{\partial}{\partial q^{i}}+(\alpha M)^{i} \frac{\partial}{\partial \dot{q}^{i}}+\eta^{\mu} \Gamma_{\mu}
$$


where $(\alpha M)^{i}:=\alpha_{k} M^{k i}$ and $\Gamma_{\mu}=\gamma_{\mu} \frac{\partial}{\partial \dot{q}^{i}}$.

It can be proven [25] that the e.o.m. $[L]=0$ is equivalent to the assertion that the dynamics is generated by $\mathbf{X}$ on the primary constraints' surface

$$
[L]=0 \Longleftrightarrow\left\{\begin{array}{c}
\mathbf{X} \\
\alpha \gamma_{\mu}=0
\end{array}\right\}
$$

\section{A.2. Proof of theorem 1}

With this preparation, the assumption that the truncation constraints, $q^{A}=0$, do not reduce the gauge freedom can be made more precise. One clear way to guarantee it is by assuming

$$
\left(\Gamma_{\mu} \dot{q}^{A}\right)_{\mathrm{R}}=0,
$$

because the requirement that these truncation constraints are preserved by the dynamics, $\left(\mathbf{X} \dot{q}^{A}\right)_{\mathrm{R}}=0$, will fix none of the arbitrary functions $\eta^{\mu}$.

Three main consequences can be drawn out of (A.6)

i) Noticing that (A.6) is just $\left(\gamma_{\mu}^{A}\right)_{\mathrm{R}}=0$, one can deduce that $\left(\gamma_{\mu}^{a}\right)_{\mathrm{R}}=\tilde{\gamma}_{\mu}^{a}$, that is, they form a basis for the null vectors of the Hessian matrix of the reduced theory. On the other hand, since $\left(\alpha_{a}\right)_{\mathrm{R}}=\tilde{\alpha}_{a}$, it follows that

$$
\left(\alpha \gamma_{\mu}\right)_{\mathrm{R}}=\tilde{\alpha} \tilde{\gamma}_{\mu},
$$

are just the primary constraints of the reduced theory.

ii) Noting that

$$
\left(\mathbf{X} \dot{q}^{A}\right)_{\mathrm{R}}=\left((\alpha M)^{A}\right)_{\mathrm{R}},
$$

we infer that the time preservation of the constraints $q^{A}=0$ requires the fulfillment of

$$
\chi^{A}:=\left((\alpha M)^{A}\right)_{\mathrm{R}}=0 .
$$

iii) Once the new constraints (A.7) are taken into account, the vector field $\mathbf{X}$ reduces to

$$
(\mathbf{X})_{\mathrm{R}}=\dot{q}^{a} \frac{\partial}{\partial q^{a}}+\left((\alpha M)^{a}\right)_{\mathrm{R}} \frac{\partial}{\partial \dot{q}^{a}}+\eta^{\mu} \tilde{\Gamma}_{\mu},
$$

where $\tilde{\Gamma}_{\mu}=\tilde{\gamma}_{\mu}^{a} \frac{\partial}{\partial \dot{q}^{a}}$. 
With the use of ii) and iii) we have the equivalence

$$
\left\{\begin{array}{c}
\mathbf{X} \\
q^{A}=0
\end{array}\right\} \Longleftrightarrow\left\{\begin{array}{c}
(\mathbf{X})_{\mathrm{R}} \\
\chi^{A}=0
\end{array}\right\}
$$

which, considering (A.5) and i), may also be written as

$$
\left([L]_{i}\right)_{\mathrm{R}}=0 \Longleftrightarrow\left\{\begin{array}{c}
\mathbf{X} \\
\alpha \gamma_{\mu}=0 \\
q^{A}=0
\end{array}\right\} \Longleftrightarrow\left\{\begin{array}{c}
(\mathbf{X})_{\mathrm{R}} \\
\tilde{\alpha} \tilde{\gamma}_{\mu}=0 \\
\chi^{A}=0
\end{array}\right\}
$$

Let us look closely at the piece $\left((\alpha M)^{a}\right)_{\mathrm{R}}$ in (A.8). Saturating again (A.3) with $\alpha_{i}$ and then reducing the result to $q^{A}=0, \dot{q}^{A}=0$, one gets, in particular,

$$
\left(W_{a b}\right)_{\mathrm{R}}(M \alpha)_{\mathrm{R}}^{b}+\left(W_{a B}\right)_{\mathrm{R}}(M \alpha)_{\mathrm{R}}^{B}=\left(\alpha_{a}\right)_{\mathrm{R}}=\tilde{\alpha}_{a}
$$

but $\left(W_{a b}\right)_{\mathrm{R}}$ is just the Hessian of the reduced Lagrangian $L_{\mathrm{R}},\left(W_{a b}\right)_{\mathrm{R}}=\tilde{W}_{a b}$, and $(M \alpha)_{\mathrm{R}}^{B}$ have been already identified as new constraints, $(M \alpha)_{\mathrm{R}}^{B} \approx 0$. All in all we have

$$
\tilde{W}_{a b}(M \alpha)_{\mathrm{R}}^{b} \approx \tilde{\alpha}_{a}
$$

which, recalling (A.4) and comparing with the e.o.m. for the reduced theory,

$$
\left[L_{\mathrm{R}}\right]_{a}=\tilde{\alpha}_{a}-\tilde{W}_{a b} \ddot{q}^{b}=0,
$$

is telling us that

$$
\ddot{q}^{b}=(M \alpha)_{\mathrm{R}}^{b}+\eta^{\mu} \tilde{\gamma}_{\mu}^{b},
$$

provided the constraints (A.7) are satisfied $\left(\eta^{\mu}\right.$ are arbitrary functions that in principle could be different from the ones used before, but they turn out to be the same). Therefore the dynamical vector field of the reduced theory can be expressed as

$$
\tilde{\mathbf{X}}=\dot{q}^{a} \frac{\partial}{\partial q^{a}}+\left((\alpha M)^{a}\right)_{\mathrm{R}} \frac{\partial}{\partial \dot{q}^{a}}+\eta^{\mu} \tilde{\Gamma}_{\mu}
$$

which coincides with $(\mathbf{X})_{\mathrm{R}}$ in (A.8). Then we have obtained the equivalence

$$
\left\{\begin{array}{c}
(\mathbf{X})_{\mathrm{R}} \\
\chi^{A}=0
\end{array}\right\} \Longleftrightarrow\left\{\begin{array}{c}
\tilde{\mathbf{X}} \\
\chi^{A}=0
\end{array}\right\}
$$

Altogether, equivalences (A.9) and (A.13) are summarized in

$$
\left\{\begin{array}{c}
\mathbf{X} \\
q^{A}=0
\end{array}\right\} \Longleftrightarrow\left\{\begin{array}{c}
\tilde{\mathbf{X}} \\
\chi^{A}=0
\end{array}\right\}
$$


But on the other hand, in a way parallel to (A.5), the reduced Lagrangian exhibits the equivalence

$$
\left[L_{\mathrm{R}}\right]_{a}=0 \Longleftrightarrow\left\{\begin{array}{c}
\tilde{\mathbf{X}} \\
\tilde{\alpha} \tilde{\gamma}_{\mu}=0
\end{array}\right\}
$$

and using both equivalences (A.14) and (A.15) together with (A.10) we get our final result

$$
\left\{\begin{array}{c}
{[L]_{i}=0} \\
q^{A}=0
\end{array}\right\} \Longleftrightarrow\left\{\begin{array}{c}
{\left[L_{\mathrm{R}}\right]_{a}=0} \\
\chi^{A}=0
\end{array}\right\}
$$

\section{A.2.1. Final REMARKS}

We shall show that the assumption (A.6) is nothing but (2.1). For the sake of simplicity we have worked with coordinates $q^{i}$ such that the holonomic constraints are written as a subset $q^{A}=0$. In a general setting the constraints will be expressed in implicit form as a set of functions

$$
f^{A}(q)=0
$$

and (A.6) will have been written as

$$
\left(\Gamma_{\mu} \dot{f}^{A}\right)_{\mathrm{R}}=0
$$

with

$$
\dot{f}^{A}=\frac{\partial f^{A}}{\partial q^{i}} \dot{q}^{i} .
$$

On the other hand it is well known [25] that a basis for the null vectors of the Hessian matrix used to define $\Gamma_{\mu}$ is provided by the gradient of the Hamiltonian primary constraints $\phi_{\mu}$ with respect to the momenta,

$$
\gamma_{\mu}^{i}=\mathcal{F} L^{*} \frac{\partial \phi_{\mu}}{\partial p_{i}},
$$

where $\mathcal{F} L^{*}$ is the pull-back of the Legendre map $\mathcal{F} L$ from tangent space to phase space. Therefore (A.17) can be written as

$$
\left(\left(\mathcal{F} L^{*} \frac{\partial \phi_{\mu}}{\partial p_{i}}\right) \frac{\partial f^{A}}{\partial q^{i}}\right)_{\mathrm{R}}=\left(\mathcal{F} L^{*}\left\{\phi_{\mu}, f^{A}\right\}\right)_{\mathrm{R}}=0,
$$

which is equivalent to

$$
\left\{\phi_{\mu}, f^{A}\right\} \mid \begin{aligned}
& \phi_{\mu}=0 \\
& f^{A}=0
\end{aligned}
$$

This is exactly (2.1). 


\section{A.3. Proof of theorem 2}

Consider that the constraints $f^{A}=0$, holonomic and non-gauge fixing, generate, via Poisson brackets, a symmetry of the equations of motion. This means that the operator $\left\{-, f^{A}\right\}$ preserves the dynamics defined by the Dirac Hamiltonian $H_{c}+\lambda^{\mu} \phi_{\mu}$ ( $H_{c}$ is the canonical Hamiltonian and $\lambda^{\mu}$ are arbitrary functions)

$$
\left\{H_{c}, f^{A}\right\}+\lambda^{\mu}\left\{\phi_{\mu}, f^{A}\right\} \approx 0,
$$

where the weak equality includes all the natural -that is, implied tby the theory itselfconstraints of the theory.

But the lhs in (A.20) is just the application of the dynamics to the constraints $f^{A}=0$ (in fact to the kinematical consequences of $f^{A}=0$, namely, $\dot{f}^{A}=0$ ). Since we already know that (A.19) holds, the constraints $\chi^{A}$ determined before can also be given an alternative expresion originated in phase space

$$
\chi^{A}=\left(\mathcal{F} L^{*}\left\{H_{c}, f^{A}\right\}\right)_{\mathrm{R}} .
$$

Considering (A.20), we infer that $\chi^{A}$ is a combination of the natural constraints of the theory, specialized to the surface $f^{A}=0$. This means that in the reduced theory it is not

necessary to introduce the constraints $\chi^{A}$, because they will automatically be included among the natural constraints exhibited by the theory. The reduced theory is then formulated without additional constraints attached and therefore the truncation is consistent.

\section{REFERENCES}

[1] For an earlier reference see

N. S. Manton, "A New Six-Dimensional Approach To The Weinberg-Salam Model," Nucl. Phys. B 158 (1979) 141.

[2] J. Scherk and J. H. Schwarz, "How To Get Masses From Extra Dimensions," Nucl. Phys. B 153 (1979) 61.

[3] M. A. MacCallum, "Anisotropic And Inhomogeneous Relativistic Cosmologies," In Hawking, S.W., Israel, W.: General Relativity, an Einstein centenary survey. Cambridge University Press, 1979, pags 533-580.

[4] J. M. Pons and P. Talavera, "Consistent and inconsistent truncations: Some results and the issue of the correct uplifting of solutions," Nucl. Phys. B 678 (2004) 427 [arXiv:hep-th/0309079]. 
[5] M. J. Duff, B. E. Nilsson and C. N. Pope, "Kaluza-Klein Supergravity," Phys. Rept. 130 (1986) 1.

[6] M. Cvetic, G. W. Gibbons, H. Lu and C. N. Pope, "Consistent group and coset reductions of the bosonic string," Class. Quant. Grav. 20 (2003) 5161 [arXiv:hep-th/0306043].

[7] H. Nastase, D. Vaman and P. van Nieuwenhuizen, "Consistency of the AdS(7) x S(4) reduction and the origin of self-duality in odd dimensions," Nucl. Phys. B 581 (2000) 179 [arXiv:hep-th/9911238].

[8] E. Witten, "Search For A Realistic Kaluza-Klein Theory," Nucl. Phys. B 186 (1981) 412.

[9] M. J. Duff, B. E. W. Nilsson and C. N. Pope, "Compactification Of D = 11 Supergravity On K(3) X T(3)," Phys. Lett. B 129 (1983) 39.

[10] J. M. Pons, "Plugging The Gauge Fixing Into The Lagrangian," Int. J. Mod. Phys. A 11 (1996) 975 [arXiv:hep-th/9510044].

[11] P. A. M. Dirac, "Lectures on Quantum Mechanics," (Yeshiva Univ. Press, New York, 1964)

[12] J. L. Anderson and P. G. Bergmann, "Constraints In Covariant Field Theories," Phys. Rev. 83 (1951) 1018.

[13] G. T. Horowitz and A. Strominger, "Black Strings And P-Branes," Nucl. Phys. B 360 (1991) 197.

[14] A. H. Chamseddine and M. S. Volkov, "Non-Abelian solitons in $\mathrm{N}=4$ gauged supergravity and leading order string theory," Phys. Rev. D 57 (1998) 6242 [arXiv:hep-th/9711181].

[15] C. V. Johnson, "D-Branes," (Cambridge University Press, 2003)

[16] Y. M. Cho and P. G. Freund, "Nonabelian Gauge Fields In Nambu-Goldstone Fields," Phys. Rev. D 12 (1975) 1711.

[17] M. A. Maldacena and C. Nunez, "Towards the large N limit of pure $\mathrm{N}=1$ super Yang Mills," Phys. Rev. Lett. 86 (2001) 588 [arXiv:hep-th/0008001]. 
[18] G. Papadopoulos and A. A. Tseytlin, "Complex geometry of conifolds and 5-brane wrapped on 2-sphere," Class. Quant. Grav. 18 (2001) 1333 [arXiv:hep-th/0012034].

[19] S. S. Gubser, A. A. Tseytlin and M. S. Volkov, "Non-Abelian 4-d black holes, wrapped 5-branes, and their dual descriptions," JHEP 0109 (2001) 017 [arXiv:hep-th/0108205].

[20] L. A. Pando Zayas and A. A. Tseytlin, "3-branes on spaces with R x S(2) x S(3) topology," Phys. Rev. D 63 (2001) 086006 [arXiv:hep-th/0101043].

[21] P. Di Vecchia, A. Lerda and P. Merlatti, " $\mathrm{N}=1$ and $\mathrm{N}=2$ super Yang-Mills theories from wrapped branes," Nucl. Phys. B 646 (2002) 43 [arXiv:hep-th/0205204].

[22] A. H. Chamseddine and M. S. Volkov, "Non-Abelian BPS monopoles in N $=4$ gauged supergravity," Phys. Rev. Lett. 79 (1997) 3343 [arXiv:hep-th/9707176].

[23] D. Z. Freedman, G. W. Gibbons and P. C. West, "Ten Into Four Won't Go," Phys. Lett. B 124 (1983) 491.

[24] M. J. Duff, B. E. W. Nilsson and C. N. Pope, "Kaluza-Klein Approach To The Heterotic String," Phys. Lett. B 163 (1985) 343.

[25] C. Batlle, J. Gomis, J. M. Pons and N. Roman, "Equivalence Between The Lagrangian And Hamiltonian Formalism For Constrained Systems," J. Math. Phys. 27 (1986) 2953. 